\title{
Topology of the polarization field in ferroelectric nanowires from first principles
}

\author{
J. W. Hong, ${ }^{1,2}$ G. Catalan, ${ }^{1}$ D. N. Fang, ${ }^{2}$ Emilio Artacho, ${ }^{1}$ and J. F. $\operatorname{Scott}^{1,3}$ \\ ${ }^{1}$ Department of Earth Sciences, University of Cambridge, Downing Street, Cambridge CB2 3EQ, UK \\ ${ }^{2} A M L$ Department of Engineering Mechanics, Tsinghua University, Beijing, 100084, P. R. China \\ ${ }^{3}$ Cavendish Laboratory, University of Cambridge, JJ Thomson Ave, Cambridge CB3 OHE, UK
}

(Dated: November 5, 2018)

\begin{abstract}
The behaviour of the cross-sectional polarization field is explored for thin nanowires of barium titanate from first-principles calculations. Topological defects of different winding numbers have been obtained, beyond the known textures in ferroelectric nanostructures. They result from the inward accommodation of the polarization patterns imposed at the surface of the wire by surface and edge effects. Close to a topological defect the polarization field orients out of the basal plane in some cases, maintaining a close to constant magnitude, whereas it virtually vanishes in other cases.
\end{abstract}

The drive towards ever smaller ferroelectric devices 1 . has resulted in novel geometries such as nanowires [2, 3], nanotubes [4, 5, 6], and nanodots [7, 8]. It was initially assumed that as the size of such nano-shapes continued to decrease, the ferroelectric polarization would vanish: ferroelectricity is a collective phenomenon after all. However, seminal works by $\mathrm{Fu}$, Bellaiche and Naumov [9, 10 suggested that, before vanishing, the polarization may first undergo a size-induced transition by which the dipoles form a ferroelectric vortex, a polar configuration that does not exist in bulk and persists down to very small diameters. This insight has triggered much work, both theoretical [11, 12, 13, 14, 15, 16 and experimental [17, 18, 19] towards establishing true critical diameters for ferroelectricity, understanding the characteristics of ferroelectric vortices, and establishing their existence.

In the present work we have used ab-initio methods to calculate the polar configuration in ultra-thin nanowires of the archetypal ferroelectric $\mathrm{BaTiO}_{3}$ (BTO). Our results show that the topological landscape of the polarization field $\vec{P}(\vec{r})$ in nanowires is much more complex than hitherto assumed, with polar configurations other than vortices appearing. The new topological textures include saddle points and quadrupoles as well as more complex configurations, all of them well described within the classic framework of topological defects of different winding numbers 20]. Such patterns are induced by the surfaces and can be understood in terms of simple surface and edge effects. Of equal importance is the discovery that the axial component of the polarization can either decrease or increase towards the surface of the wire depending on its termination, thus resolving the longstanding controversy on the sign of the so-called extrapolation length $21,22,23$. More importantly, the critical size for the persistence of polarization in nanowires is found to be determined by their surfaces.

The two-dimensional (2D) topology of of the polarization field in thin nanostructures can be mapped onto the topology of $\left(P_{x}, P_{y}\right)$ for wires if the field remains constant along $z$, the axial direction of the wire. This paper focuses on topological defects in such 2D field [20] (these so-called "defects" do not refer to structural defects of the wires). The winding number is defined as $n=\phi / 2 \pi$, being $\phi$ the total angle the 2D field vector rotates when going around a closed circuit. For a continuous field $n$ must be an integer, which if different from zero implies the existence of at least one defect within the circuit. Any smooth deformation of a vector field will conserve $n$, while changing the winding number implies a dramatic, extended and highly energetic rearrangement 33. Such $\Delta n \neq 0$ rearrangements have been used to define "phase transformations" 24 for nano-objects displaying vortices $(n=+1)$. Here we find realizations of $n=0,1,-1$, and -3 . We see textures that were described before (e.g. radial 25]) together with completely novel ones. The different textures are induced (and thus can be manipulated) by the different surface terminations of the wires.

Our calculations were performed within DFT and the generalized gradient approximation (GGA) [26]. We used the Siesta method [27, 28, based on finite-range numerical atomic orbitals, using a double- $\zeta$ polarized basis set 11, 29. Norm-conserving pseudopotentials were used, including into the valence the semicore shells $3 s$ and $3 p$ for $\mathrm{Ti}$ and $5 s$ and $5 p$ for Ba. The performance of the method was tested for bulk $\mathrm{BaTiO}_{3}$. The obtained lattice parameter for the rhombohedral phase is $0.12 \%$ smaller than the experimental value of $4.0036 \AA$ at $15 \mathrm{~K}$ [30], and $0.055 \%$ smaller than the published results with the same GGA and plane waves as basis set [26]. The rhombohedral angle was found to be $89.84^{\circ}$, exactly as obtained in the same experiments, and the off-centering of the $\mathrm{Ti}$ atom came out as $0.184 \AA$, as compared with the experimental $0.185 \AA$. Periodic boundary conditions were used with a simulation cell describing a square array of infinite nanowires, such that periodic replicas are separated by $24 \AA$. The width of the wires is shown in Fig. 1. The unit cell along the wire axis comprises a single bilayer in all cases, and therefore all polarization textures are homogeneous along $z$ by construction. Integrals in reciprocal space used a $\vec{k}$-mesh of $10 \AA$ cutoff [31], while the integrals in real space used a $\vec{r}$-mesh of 350 Ry cutoff [28]. The atomic positions were relaxed until all atomic force components were smaller than $10 \mathrm{meV} / \AA$. From the re- 
(a)
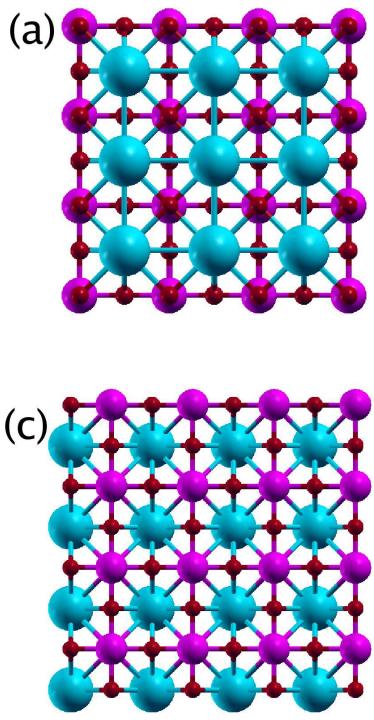

(b)

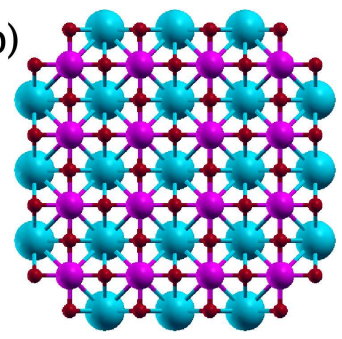

(d)

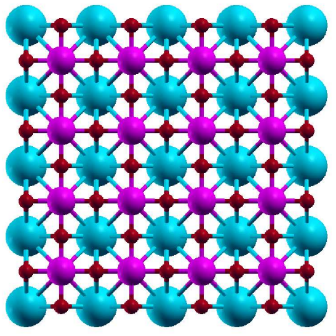

FIG. 1: (Color online) Sections of the different $\mathrm{BaTiO}_{3}$ nanowires considered. The largest balls (cyan) are for Ba ions, the intermediate ones (magenta) represent $\mathrm{Ti}$ ions, and the smallest ones (red) are for O. (a) is $\mathrm{TiO}_{2}$ terminated, (b) is $\mathrm{BaO}$ terminated, except the edges, where lines of $\mathrm{Ba}$ atoms have been removed, (c) is stoichiometric, with two $\mathrm{BaO}$ surfaces and two $\mathrm{TiO}_{2}$ ones. (d) is $\mathrm{BaO}$ terminated.

laxed structures, the Ti displacement from the centre of its coordination octahedron was taken to define $\vec{P}\left(\vec{r}_{\mathrm{Ti}}\right)$, as a measure of the local dipole. For Ti atoms on surfaces or edges the off-center displacement was determined by the remaining coordinating $\mathrm{O}$ atoms. It should be emphasized, however, that the focus of the paper is on the topological aspects of the polarization, which are not affected by quantitative details related to this definition.

Four different kinds of nanowires have been considered, corresponding to different surface terminations, as shown in Fig. 1, all showing (100) and (010) surfaces, or slight alterations thereof. Two of them display the same termination on all sides, namely, all $\mathrm{TiO}_{2}$-terminated (a), or all BaO-terminated (d). Wire-type (c) is stoichiometric, with both terminations, and corresponding to the wires studied in ref. [11. Finally (b) is like (d) except for the fact that the $\mathrm{Ba}$ on the edges has been removed. Each wire type has been considered in several sizes, mainly $4 \times 4$, and $5 \times 5$, counting the Ti atoms.

Fig. 2 shows the polarization textures of the wire types of Fig. 1, alongside qualitative sketches of the corresponding topological defects for a $2 \mathrm{D}$ vector field. The discrete set of off-centering vectors is assimilated to a lattice discretization of a $2 \mathrm{D} \vec{P}$ field. Different wire widths correspond to different discretizations of the corresponding general pattern. A first striking result is that even for the small sizes considered (and the coarse discretization they imply) the field textures appear very clearly. All textures

preserve inversion symmetry at the center of the wire if it was not previously broken by the surface termination. The first two cases in Fig. 2, displaying inward and outward radial patterns, correspond to the same kind of topological defect, of winding number 1 . They are thus homotopical with each other, but also with the vortex structures seen in refs. [10, 13]. The continuous transformation taking a radial texture onto a vortex one is beautifully illustrated in Mermin's review [20. They of course differ in other physical aspects, such as the nonzero toroidal moment of the vortex patterns.

Figs. 2(c) and (d) correspond to different winding numbers as yet not observed or proposed in this field, namely -1 and -3 . In Fig. 2(c) the defect is displaced from the center (it is the only non-centrosymmetric wire). Fig. 2(d) shows the $n=-3$ case of the $\mathrm{BaO}$ terminated wire. The high winding number imposes a more rapidly varying field around the defect, which implies higher energy. The system responds by a more pronounced depression of the magnitude of the 2D field around the defect. The winding of the field around the outer circuit in the wire section clearly corresponds to $n=-3$.

Fig. 2(e) is for the same $\mathrm{BaO}$ termination as Fig. 2(d) but smaller thickness. Although a different discretization, the winding around the outer circuit clearly shows the same $n=-3$. In this case the inner displacements, although small, all point mainly outwards, inconsistently with the the $n=-3$ texture: This system has found more stability in decomposing the global $n=-3$ global defect into a set of defects whose winding numbers add up to -3 . In general 20], when following the winding of the field around a circuit the winding number obtained is the sum of winding numbers of all the point defects enclosed in the circuit. In this case the texture can be described by a central defect with $n=+1$, the field radiating outward as in Fig. 2(g), plus four other defects of $n=-1$, as illustrated in the last sketch.

The described textures are clearly induced by surface and edge effects. Indeed, the phenomenlogy described can be understood as originated by three simple tendencies: (i) In Ti terminated surfaces and edges, the Ti atoms tend to off-center inwards, (ii) Ba surface termination induces outward $\mathrm{Ti}$ off-centering patterns, while (iii) Ba edge termination induces an inward tendency. The competition of $(i i)$ and (iii) in the Ba terminated wires gives rise to the rich $n=-3$ pattern. By removing the Ba edges [Fig. 2(b)], the competition disappears and the fully outward pattern is established. Similarly, when removing the $\mathrm{Ba}$ edge in the stoichiometric case the polarization field becomes topologically homogeneous $(n=0)$, with the field lines crossing the wire diagonally pointing left and down (not shown). Special chemical tendencies at edges and surfaces have been previously reported [16, 17]. Shimada et al. [16] talks specifically about strong "edge bonds" for $\mathrm{PbTiO}_{3}$ nanowires.

The surfaces not only determine the $\left(P_{x}, P_{y}\right)$ topology, 
(a)

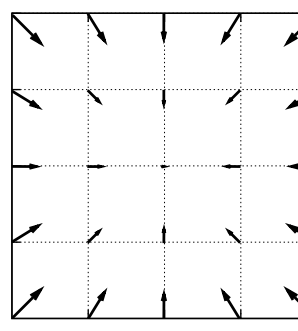

(b)

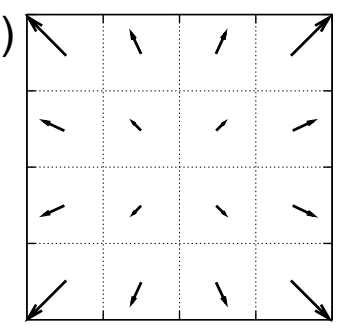

(c)

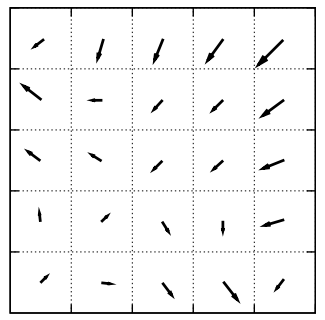

(d)

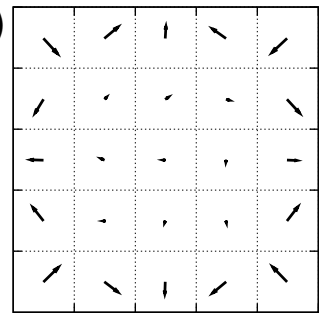

(e)

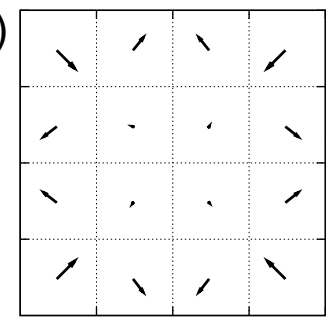

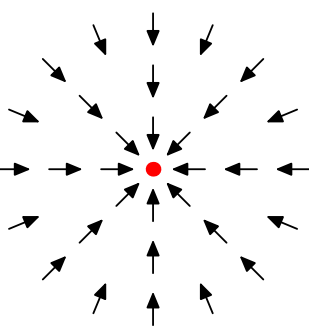

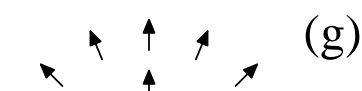

$(\mathrm{g})$
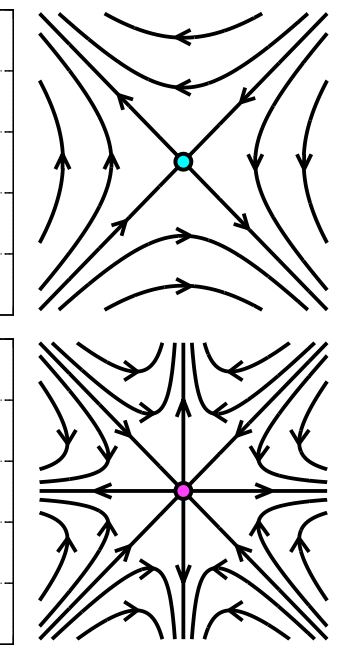

(i)

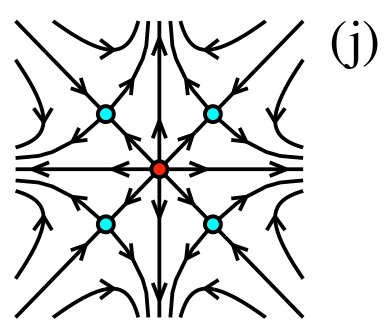

FIG. 2: Left column (a)-(d): Ti off-center displacements for the four respective nanowires in Fig. 1. (e) is analogous to (d) but for a thinner wire. The right column sketches the corresponding $2 \mathrm{D}$ field lines around topological point defects of winding numbers $n=1,1,-1$, and -3 , respectively, (j) showing the $n=-3$ decomposition into a central $n=+1$ and four $n=-1$ defects. The respective largest arrows corresponds to (a) $21 \mathrm{pm}$, (b) $35 \mathrm{pm}$, (c) $23 \mathrm{pm}$, (d) $6.8 \mathrm{pm}$, and (e) $6.6 \mathrm{pm}$.

but also the $P_{z}$ behaviour (Fig. 3). Axial switchable polarization has been observed experimentally [18, and has been analyzed within Landau-Ginzburg theory [12, 14], and DFT [11, 16]. We find that for $\mathrm{TiO}_{2}$ termination the $4 \times 4$ wires display $P_{z}=0$, while it is finite for $5 \times 5$ (in the figure) or larger. When non-zero, the axial polar-

ization stems mainly from the surface cells, the central cells displaying much smaller displacements. This is surprising: an essentially constant-magnitude field is commonly assumed for these and other ferroelectric systems (some models have this assumption built in, as a constant dipole moment of arbitrary orientation). Here, however, the field responds by vanishing smoothly into the defect. The $\mathrm{BaO}$ terminated wires respond in the more expected way: discontinuity at the defect is avoided without substantial diminishing of the polarization field, by having maximal axial polarization at the wire center. This is the case for both with and without Ba edges, although the latter shows a much smaller axial component at the center. There is also a $3 \times 3$ critical thickness for $P_{z}$ (largest system with $\left.P_{z}=0\right)$ in the Ba terminated case. In the stoichiometric wires the situation is different. The figure shows a $5 \times 5$ case (the critical thickness for this type is $2 \times 2$, in agreement with ref. [11]), which again shows large $P_{z}$ for the surface and edge Ti displacements, for which the in plane components were also large.

In addition to their instrinsic fundamental interest, the polarization-field textures and the possibility of manipulating them, offer interesting exploitation possibilities. Net polarization has been measured and switched perpendicularly to the wire axis at room temperature with a scanning probe microscope [2, 3, 17]. Such a setup should thus respond as a three-state system, with $+1,0$ and -1 states, the +1 and -1 corresponding to the conventional ferroelectric response, and the zero to the stable topologically defective state (with $|P|$ much smaller than for the other states, strictly zero if the unpolarized wire is centrosymmetric). The field rearrangement needed for a non-homotopical change of texture would mean an energy barrier when changing the net polarization, giving a scenario analogous to a first order phase transition above the transition temperature: the global minimum for $P \sim 0$ and two local minima for + and -. Note that some of the $n \neq 0$ textures exhibit net quadrupoles. These configurations can be switched (destabilized) by application of an electric field gradient, which could in principle be used for a quadrupole memory device.

Phase transformations were already proposed for vortex structures in nanodots [10]. In wires, as extended systems, the expression would be more appropriate except for the fact that wires with sufficiently short-ranged interactions do not sustain spontaneous symmetry breaking at any finite temperature 32. Even assuming that the long range dipole-dipole interactions and the also long-ranged strain mediated interactions do not invalidate the previous argument, the discussions in this paper can be understood in terms of the observed wires being of lengths smaller that the dipole-dipole correlation length: any perturbation pins the order parameter (the polarization across wires is experimentally observed after all). As a final remark, the surface and edge control not only affects the polarization across the wires, but can 

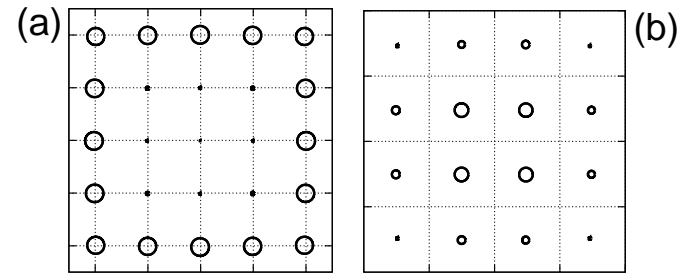

(c)
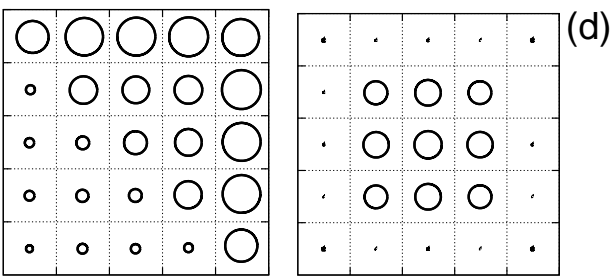

FIG. 3: $z$ component of the Ti off-centering for the wires in Fig. 1. The largest circle represents a value of $15 \mathrm{pm}$.

also determine the conductance along the wire, since the deviations from $(\mathrm{BaO})_{x}\left(\mathrm{TiO}_{2}\right)_{y}$ compositions give rise to doped wires, opening still further possibilities for these systems, to be explored elsewhere. Evidently, different surface chemistries than the ones contemplated here can be achieved depending on how the wires are produced. The points remain, however, that interesting topologies are to be expected in thin ferroelectric wires, and that they are induced by the surfaces and edges.

In summary, based on first-principles calculations, we predict ferroelectric patterns in BTO nanowires for different surface terminations, finding new textures that correspond to the topological winding numbers $n=0,+1,-1$ and -3 of point defects in $2 \mathrm{D}$ vector fields. A tendency of the $n=-3$ defect to decompose into one +1 and four -1 defects is observed, as well as the different mechanisms used by the field to avoid discontinutities.

J. W. Hong thanks China's Scholarship Council for its support. We acknowledge support through EPSRC and the computing resources of Cambridge's CamGRID and the High Performance Computing Service.

[1] J. F. Scott, Ferroelectric memories, Springer, Berlin, 2000. ISBN: 978-3-540-66387-4.

[2] J. J. Urban, W. S. Yun, Q. Hu, and H. Park, J. Am. Chem. Soc. 124, 1186 (2002).

[3] W. S. Yun, J. J. Urban, Q. Hu, and H. Park, Nano Lett. 2, 447 (2002).

[4] Y. Luo, I. Szafraniak, N. D. Zakharov, V. Nagarajan, M. Steinhart, R. B. Wehrspohn, J. H. Wendorff, R. Ramesh, and M. Alexe, Appl. Phys. Lett. 83, 440 (2003).

[5] F. D. Morrison, L. Ramsay, and J. F. Scott, J. Phys. Condens. Matter 15, L527 (2003).

[6] Y. Mao, S. Banerjee, and S. S. Wong, Chem. Commun. 3, 408-409 (2003).
[7] H. J. Shin, J. H. Choi, H. J. Yang, Y. D. Park, Y. Kuk, and C. J. Kang, Appl. Phys. Lett. 87, 113114 (2005).

[8] M.-W. Chu, I. Szafraniak, R. Scholz, C. Harnagea, D. Hesse, M. Alexe, and U. Gösele, Nature Materials 3, 8790 (2004).

[9] H. Fu and L. Bellaiche, Phys. Rev. Lett. 91, 257601 (2003).

[10] I. I. Naumov, L. Bellaiche and H. Fu, Nature 432, 737 (2004).

[11] G. Geneste, E. Bousquet, J. Junquera and P. Ghosez, Appl. Phys. Lett. 88, 112906 (2006).

[12] A. N. Morozovska, E. A. Eliseev, and M. D. Glinchuk, Phys. Rev. B 73, 214106 (2006).

[13] S. Prosandeev, I. Ponomareva, I. Naumov, I. Kornev and L. Bellaiche, J. Phys. Condens. Matter 20, 193201 (2008).

[14] J. W. Hong and D. Fang, J. Appl. Phys. 104, 064118 (2008).

[15] J. Wang and M. Kamlah, Appl. Phys. Lett 93, 262904 (2008).

[16] T. Shimada, S. Tomoda, and T. Kitamura, Phys. Rev. B 79, 024102 (2009).

[17] J. E. Spanier, A. M. Kolpak, J. J. Urban, I. Grinberg, L. Ouyang, W. S. Yun, A. M. Rappe, and H. Park, Nano Lett. 6, 735 (2006).

[18] Z. Y. Wang, A. P. Suryavanshi, and M.-F. Yua, Appl. Phys. Lett. 89, 082903 (2006).

[19] J. B. Rodriguez, X. S. Gao, L. F. Liu, W. Lee, I. I. Naumov, A. M. Bratkovsky, D. Hesse, and M. Alexe, Nano Letters 9, 1127 (2009).

[20] N. D. Mermin, Rev. Mod. Phys. 51,591 (1979).

[21] R. Kretschmer and K. Binder, Phys. Rev. B 20, 1065 (1979).

[22] W. L. Zhong, Y. G. Wang, P. L. Zhang, and B. D. Qu , Phys. Rev. B 50, 698 (1994),

[23] A. M. Musleh, L.-H. Ong, and D. R. Tilley, J. Appl. Phys. 105, 061602 (2009).

[24] I. Naumov and H. Fu, Phys. Rev. Lett. 98,077603 (2007).

[25] J. W. Hong and D. Fang, Appl. Phys. Lett. 92, 012906 (2008).

[26] Z. Wu and R. E. Cohen, Phys. Rev. B 73, 235116 (2006).

[27] P. Ordejon, E. Artacho, and J. M. Soler, Phys. Rev. B 53, R10441 (1996).

[28] J. M. Soler et al., J. Phys. Condens. Matter 14, 2745 (2002).

[29] E. Anglada, J. M. Soler, J. Junquera, and E. Artacho, Phys. Rev. B 66, 205101 (2002).

[30] G. H. Kwei, C. Lawson, and S. J. L. Billinge, J. Phys. Chem. 97, 2368 (1993).

[31] J. Moreno and J. M. Soler, Phys. Rev. B 45, 13891 (1992).

[32] N. D. Mermin and H. Wagner, Phys. Rev. Lett. 17, 1133 (1966).

[33] Strictly speaking, the topological defects mentioned here arise for vector fields of constant magnitude, giving rise to a discontinuity in the field derivatives at the defect. Ferroelectric fields (or their proxies, e.g. cation off-centerings) are of variable magnitude and vanish at the defect. 